\title{
Las víctimas invisibles de la Violencia de Género
}

\author{
María del Prado Ordóñez Fernández ${ }^{a}$ Patricio González Sánchez ${ }^{b}$
}

\begin{abstract}
a Médico Psiquiatra de la Unidad de Salud Mental del Hospital General de Ciudad Real.

${ }^{\mathrm{b}}$ Fiscal Sustituto. Fiscalía, Tribunal Superior de Justicia, Madrid.

\section{Correspondencia:}

Ma del Prado Ordóñez

Fernández,

C/ Alarcos $n^{\circ} 3,1^{\circ} \mathrm{D}, 13001$ Ciudad Real, España. E-mail: mpordonez@sescam.jccm.es
\end{abstract}

Recibido el 11 de julio de 2011.

Aceptado para su publicación el 1 de septiembre de 2011.

\begin{abstract}
RESUMEN
El objetivo de esta revisión es sensibilizar a todos los profesionales en contacto con la infancia (salud, educación, policía, servicios sociales, puntos de encuentro) del problema del impacto que este tipo de violencia tiene sobre las niñas y niños que viven en estos hogares y que, generalmente, comparten estas situaciones de violencia, directa o indirecta, con sus madres, o aquellas figuras femeninas que desempeñan el rol de madres y de las consecuencias de este maltrato en el desarrollo del niño en todas las esferas: física, cognitiva, afectiva, social y emocional.
\end{abstract}

Palabras clave. Niño, Violencia contra la Mujer.

\section{ABSTRACT}

The invisible victims of Gender Violence

The aim of this review is to increase awareness amongst all professionals dealing with children (in areas of health, education, police, social services, etc.) of the negative impact that violence against women has on them. Children from these homes generally share situations of violence, either directly or indirectly, with their mothers or with female figures who take on the role of mothers. The consequences of this form of abuse affect child development in all aspects: physical, cognitive, emotional and social.

Key words. Child, Violence Against Women.

\section{INTRODUCCIÓN}

En las últimas décadas el problema de la violencia intrafamiliar hacia las mujeres ha acaparado el interés de un buen número de investigaciones, que han centrado sus estudios en las repercusiones que el comportamiento de los agresores tiene sobre la salud física y psicológica de sus víctimas. De ahí el gran número de publicaciones recientes que sobre los más variados aspectos de las consecuencias de la violencia hacia las mujeres han aparecido tanto a nivel nacional como internacional ${ }^{1}$.

Sin embargo, son mucho más escasos los estudios sistemáticos en los que se analiza el impacto que este tipo de violencia tiene sobre las niñas y niños que viven en estos hogares y que, generalmente, comparten estas situaciones de violencia, directa o indirecta, con sus madres, o aquellas figuras femeninas que desempeñan el rol de madres. En estos casos, a la ya compleja problemática que supone ser víctima de agresiones, de tipo físico y verbal, en la edad adulta habría que añadir las tremendas repercusiones negativas que vivir este tipo de situaciones tiene sobre el desarrollo de los miembros más jóvenes de la familia. Repercusiones que se manifiestan en todas y cada una de las esferas del desarrollo humano: físico, cognitivo, afectivo, social y emocional, y que se interrelacionan mutuamente para producir importantes problemas en los sujetos que las padecen ${ }^{1}$. 


\section{VIOLENCIA FAMILIAR}

El término violencia familiar hace referencia a cualquier forma de abuso, ya sea físico, psicológico o sexual, que tiene lugar entre los miembros de una familia ${ }^{2}$. Como todo abuso, implica un desequilibrio de poder, y es ejercido desde el más fuerte hacia el más débil con el fin último de conseguir un control sobre la relación. Tradicionalmente, en nuestra sociedad, dentro de la estructura familiar jerárquica actualmente predominante, los dos principales ejes de desequilibrio los han constituido el género y la edad, siendo las mujeres, los niños y los ancianos las principales víctimas de la violencia dentro de la familia ${ }^{3}$.

La familia como institución se ha considerado, históricamente, un ámbito privado donde el comportamiento de sus miembros se situaba fuera del control social. Las creencias y mitos culturales asociados al sistema patriarcal han legitimado desde tiempos remotos el poder y la dominación del marido hacia la mujer y los hijos, despojando a éstos de todo derecho legal, económico o social ${ }^{4}$. Tanto la mujer como sus hijos carecían de individualidad, absorbidos por la del hombre cabeza de familia, a cargo de quien legalmente estaban y tenía plenos derechos para usar las medidas que creyera convenientes para mantener el control sobre ellos ${ }^{3}$.

Actualmente puede resultar difícil aceptar, debido a determinados estereotipos ideales del concepto de familia (agente socializador básico, garante de seguridad, apoyo y afectos), que la familia es uno de los grupos sociales en los que se dan más comportamientos violentos. Straus y Gelles, basándose en los resultados de una de las encuestas de victimización más amplias realizadas hasta entonces en población norteamericana, afirmaron que es más probable que una persona sea golpeada o asesinada en su propio hogar por otro miembro de su familia que en ningún otro sitio o por ninguna otra persona ${ }^{3}$. En nuestro país, según estimaciones del Ministerio del Interior, un tercio del total de homicidios cometidos anualmente tienen como víctima y victimario a miembros de una misma familia y alrededor de una cuarta parte de las denuncias de delitos y faltas de lesiones presentadas en dependencias policiales se producen en el ámbito familiar ${ }^{3}$. A las estimaciones oficiales, ya de por sí considerables, habría que añadir los casos que conforman la llamada cifra negra, casos de violencia física o psicológica que ocurren dentro del contexto familiar no denunciados $y$, por tanto, ocultos a las estadísticas ${ }^{3}$.
Según Straus y Gelles ${ }^{3}$, uno de los factores más relevantes a la hora de explicar la elevada incidencia de la violencia familiar es el hecho de que la familia posee una serie de características que la hacen potencialmente conflictiva, con el correspondiente riesgo de que los conflictos puedan resolverse de manera violenta.

Entre estas características destacan:

a) La alta intensidad de la relación, determinada por la gran cantidad de tiempo compartido entre sus miembros, el alto grado de confianza entre ellos, el derecho a influir sobre los demás y el elevado conocimiento mutuo que se deriva de la convivencia diaria.

b) La propia composición familiar, integrada por personas de diferente sexo y edad, lo que implica la asunción de diferentes roles a desempeñar, y que se traduce en unas marcadas diferencias de motivaciones, intereses y actividades entre sus miembros.

c) El alto nivel de estrés al cual está expuesta la familia como grupo, debiendo hacer frente a distintos cambios a lo largo del ciclo vital y a exigencias de tipo económico, social, o laboral.

d) El carácter privado que posee todo aquello que ocurre en el interior de una familia y que, tradicionalmente, la ha hecho situarse fuera del control social.

Por otra parte, en las familias en donde se ejerce la violencia, se presentan formas jerárquicas de organización, con funciones rígidamente definidas que colocan a la mujer en una situación de subordinación. Estas familias se caracterizan por su rigidez y resistencia al cambio, y en ellas la violencia es una condición y un resultado para mantener este tipo de estructura de dominación ${ }^{3}$.

La violencia que tiene lugar en la familia y en la sociedad es parte de un mismo sistema. Así, en la medida en que las relaciones de poder que se establecen en la familia incluyen el uso de la violencia, se condiciona a los individuos a aceptar la violencia como método legítimo para imponer la autoridad en este y otros estamentos sociales. Paralelamente, la legitimidad de pautas violentas en la sociedad avala la presencia de este tipo de interacciones en la familia ${ }^{3}$.

Según diferentes estudios, se estima que en torno al $60-70 \%$ de los niños, niñas y adolescentes que viven en hogares violentos son víctimas más o menos directas de situaciones de maltrato. Asimismo, en los últimos diez años se han constatado en nuestro 
país algunos datos realmente preocupantes entre los que cabe señalar, a título de ejemplo, los dos siguientes. El primero hace referencia al hecho de que se han contabilizado, al menos, 53 casos de niñas y niños asesinados por sus progenitores varones. Dichos asesinatos, en la mayoría de los casos, tuvieron lugar durante el cumplimiento del régimen de visitas establecido en la sentencia de separación o divorcio. El segundo dato es el procedente del número de menores de edad secuestrados por sus progenitores que no tienen su guarda y custodia y que asciende a un total de, aproximadamente, $150^{1}$.

\section{CONSECUENCIAS DE LA EXPOSICIÓN DE LOS NIÑOS A LA VIOLENCIA FAMILIAR}

La exposición a la violencia familiar constituye un grave riesgo para el bienestar psicológico de los menores, especialmente si, además de ser testigos, también han sido víctimas de ella.

Resultados hallados en diversos estudios muestran que los niños expuestos a la violencia en la familia presentan más conductas agresivas y antisociales (conductas externalizantes) y más conductas de inhibición y miedo (conductas internalizantes) que los niños que no sufrieron tal exposición ${ }^{3}$. Los niños de estos hogares violentos también suelen presentar una menor competencia social y un menor rendimiento académico que los niños de familias no violentas ${ }^{3}$, además de promedios más altos en medidas de ansiedad, depresión y síntomas traumáticos ${ }^{3}$. Se estima que entre el 25 y el $70 \%$ de los niños de familias en las que se producen episodios de violencia manifiestan problemas clínicos de conducta, especialmente problemas externos como conductas agresivas y antisociales ${ }^{3}$.

Similares conclusiones se extraen de un estudio llevado a cabo en nuestro país por Corbalán y Patró ${ }^{5}$, quienes administraron un cuestionario específico a una muestra de 40 mujeres maltratadas, residentes en centros de acogida, sobre la existencia de alguna forma de maltrato de su pareja hacia sus hijos y sobre los principales síntomas y comportamientos problemáticos apreciados en ellos. Los resultados de este estudio arrojaron los siguientes datos sobre sintomatología presente en los hijos de las mujeres de la muestra: el $10 \%$ presentaron conflictividad en la escuela; el 7,5\% huyeron del hogar; el 35\% mostraron comportamientos violentos entre iguales; el $22,5 \%$ tuvieron comportamientos violentos hacia la madre; el $25 \%$ bajo rendimiento escolar; el $27,5 \%$ miedo hacia el maltratador; el 32,5\% síntomas de ansiedad y el $30 \%$ tristeza y aislamiento.

\section{EFECTOS DE LA VIOLENCIA DE GÉNERO EN LA INFANCIA}

Según Espinosa Bayal ${ }^{1}$, hay una serie de consecuencias comunes tanto a la exposición directa como indirecta a la violencia. Las principales consecuencias en la infancia y en la adolescencia de la exposición directa e indirecta a situaciones de violencia intrafamiliar se pueden resumir como sigue:

$1^{\circ}$.- Exposición directa:

-Consecuencias físicas: retraso en el crecimiento, alteraciones del sueño y de la alimentación, retraso en el desarrollo motor, etc.

-Alteraciones emocionales: ansiedad, depresión, baja autoestima, trastorno por estrés posttraumático, etc.

-Problemas cognitivos: retraso en el lenguaje, absentismo escolar, fracaso escolar, etc.

-Problemas de conducta: falta de habilidades sociales, agresividad, inmadurez, delincuencia, toxicomanía, etc.

\section{$2^{\circ}$.- Exposición indirecta:}

- Incapacidades de las madres para atender las necesidades básicas de los niños y niñas, por la situación física y emocional en la que se encuentran, lo que puede generar situaciones de negligencia y abandono.

-Incapacidades de los agresores para establecer una relación cálida y afectuosa cercana con sus hijos e hijas, lo que puede generar serios problemas de vinculación afectiva y de relaciones de apego.

Pedreira Masa describe, en su teoría del "Círculo Interactivo de la Violencia Familiar"6, que la mujer víctima, como consecuencia del maltrato por parte de su pareja, padece una alta tasa de estrés, lo que puede generar un vínculo con su hijo en el que predomine este factor. Igualmente puede padecer síntomas psicológicos y físicos tales como angustia, trastornos depresivos, trastornos somatomorfos y descompensaciones de procesos crónicos como diabetes o hipertensión arterial, que puede alterar negativamente sus habilidades de manejo eficaz con los hijos. Éstos, a su vez, también pueden estar expuestos a elevadas tasas de estrés, apareciendo o incrementándose en ellos síntomas y comportamientos consistentes en irritabilidad, trastornos afectivos, trastornos somatoformos, descargas agresivas, oposicionismo, etc. Estas 
alteraciones, tanto en la madre como en los hijos, serán fuente de tensión y afectarán negativamente al vínculo materno-filial.

\section{CARACTERÍSTICAS DEL DESARROLLO PSICO- LÓGICO DE LAS NIÑAS Y NIÑOS VÍCTIMAS DE VIOLENCIA INTRAFAMILIAR}

Resulta muy complicado hablar, en general, de consecuencias de la violencia intrafamiliar en el desarrollo psicológico de niños y niñas. Existen numerosas variables cuya influencia puede afectar, $y$ de hecho afectan, a dichas consecuencias ${ }^{1}$.

Entre estas variables cabe señalar, básicamente, las siguientes: la mayor o menor cantidad de tiempo que el niño o la niña están expuestos a las situaciones violentas; el tipo de violencia que sufren (física, emocional, directa, indirecta, etc.); la edad del menor que se encuentra expuesto a las situaciones de violencia intrafamiliar; la relación del agresor con la víctima, y por tanto con la "víctima de la víctima"; la posibilidad de recibir, o no, ayuda especializada ${ }^{1}$.

Todas estas variables, y algunas otras derivadas de ellas, hacen que las consecuencias sean más o menos graves. No obstante, y a pesar de las dificultades existentes, se puede hacer una caracterización más o menos general de las consecuencias que tiene vivir en un entorno familiar violento para el desarrollo psicológico de sus miembros más jóvenes.

Un resumen de tales consecuencias se expone a continuación:

$1^{\circ}$.- En el desarrollo social, las principales consecuencias son:

-Dificultades de interacción social.

-Problemas de agresividad.

-Problemas de inhibición.

-Dificultades para interpretar las claves sociales.

-Falta de habilidades de resolución de problemas sociales.

-Tendencia a interpretar de forma hostil la conducta de los otros.

$2^{\circ}$.- En el campo del desarrollo emocional cabe destacar:

-Falta de empatía.

-Dificultades para expresar y comprender

emociones, tanto propias como ajenas.

-Problemas de autocontrol de la propia conducta. $3^{\circ}$.- En el ámbito del desarrollo cognitivos, las principales dificultades son:

-Baja autoestima.

-Indefensión aprendida.

-Tendencia a no enfrentarse a nuevas tareas por miedo al fracaso y a la frustración.

-Problemas de egocentrismo cognitivo y social.

-Juicios morales heterónomos: más permisivos con sus transgresiones que con las de los demás.

Todas estas consecuencias psicológicas van a influir en la integración escolar del niño, produciendo desajuste escolar. Este desajuste se traduce en problemas de rendimiento académico, absentismo escolar, falta de motivación, atención y concentración, hechos que asociados a la escasez de habilidades sociales pueden convertirles en "dobles víctimas", ya que a los problemas que sufren en su entorno familiar se pueden sumar problemas en el entorno escolar: acoso, aislamiento, etc ${ }^{1}$.

De cualquier manera, las repercusiones psicológicas de la violencia familiar padecida por niños dependen de varios factores:

a) Características personales de la víctima: edad, desarrollo, vulnerabilidad, padecimiento de alguna discapacidad.

b) Las circunstancias del maltrato: cronicidad, tipología, gravedad, severidad de éste.

c) La protección que ejerza la figura materna o el entorno familiar sobre el menor.

\section{IMPACTO DE LA VICTIMIZACIÓN SEGÚN LAS DIFERENTES ETAPAS EVOLUTIVAS}

Finkelhor $^{3}$, basándose en la victimología evolutiva, diferencia la respuesta a la victimización en función de las diferentes etapas evolutivas del desarrollo infantil.

El grado de expresión y verbalización de los pensamientos y emociones depende en los niños de la etapa de su desarrollo, de las características de su personalidad y de la relación familiar y social.

Ya en el embarazo, la madre puede sufrir malos tratos físicos o psicológicos, pudiendo marcar, a veces, el momento en el que se recrudezca la violencia ejercida sobre ella por su pareja. Las consecuencias que de ello se pueden derivar son: parto prematuro, bajo peso al nacer, mortalidad perinatal, menor seguimiento del embarazo, mayor grado de interrupciones no voluntarias del mismo y menor participación y preparación para el parto, 
además de un incremento en el consumo de alcohol y ansiolíticos por la madre.

Durante la primera infancia, los niños que experimentan malos tratos pueden crecer con una falta o una desorganización en el apego, pudiendo presentar trastornos de relación con sus iguales, conductas de retraimiento y retrasos cognitivos. En sus primeros dos años de vida, los menores perciben el miedo y la ansiedad de sus madres $y$, como pueden ser ignoradas sus necesidades, esto les conduce a situaciones de desconfianza y de abandono emocional. Desde los dos a los cinco años, los menores observan la realidad sin comprenderla, a los que se añade la dificultad para diferenciar la fantasía de la realidad, pudiendo llegar a creer que son ellos la razón del conflicto, instalándose en ellos sentimientos de culpa, inutilidad y ansiedad. La sintomatología principal en esta etapa comprende: miedo, sentimiento de desamparo e impotencia, creencia de que pueden morir durante las agresiones, ansiedad, inseguridad, dudas, expectación, actitudes de negación y de regresión, tristeza y aislamiento.

En la infancia media, los problemas afectan, fundamentalmente, a su desarrollo socio-emocional. Las dificultades de comprensión y asimilación de los problemas se expresa a través de sintomatología de ansiedad, depresión y cognición (fantasías). A medida que el niño crece y aumenta su capacidad para comprender y asimilar la realidad, se pueden presentar alianzas con uno de los progenitores, culpabilizando al otro, lo que sienta las bases de la identificación de roles.

En la etapa preadolescente, los sentimientos de frustración y desamparo se pueden traducir en comportamientos violentos o antisociales y mala conducta escolar. Algunos son provocadores y agresivos para adquirir sensación de poder, otros son evitadores de relaciones, según se identifiquen con el agresor o con la víctima. En ocasiones pueden adoptar posiciones prematuramente adultas de protección a sus madres y hermanos.

En la adolescencia, se alcanza el desarrollo intelectual, pero es asimétrico, dado que la mayor capacidad de análisis y evaluación no va paralela a los conocimientos y experiencias que presenta el sujeto adulto, por lo que se pueden idear soluciones utópicas, presentar salidas en falso, etc. Además, es una época en la que se abordan temáticas propias del ámbito de la personalidad, como es la identidad personal, rol sexual, competencia personal, plan- teamiento de futuro, intereses, valores, conducta, etc., por lo que la vivencia del maltrato a su madre puede suponer un punto importante de desequilibrio en su desarrollo integral. Los síntomas más frecuentes durante esta etapa están relacionados con actitudes de responsabilidad excesiva en el hogar, pueden adquirir un papel de protector activo, pudiendo interponerse entre la madre y su agresor, pudiendo llegar a recibir los mismos golpes, e incluso llegando, ellos mismos, a agredir a sus propios padres. También se presentan bajos niveles de autoestima, altos niveles de ansiedad y depresión y problemas académicos. También pueden adoptar cambios radicales en sus estilos de vida o evadirse mediante el sexo o la delincuencia. Ocasionalmente se produce embotamiento emocional, frialdad e indiferencia.

Así, de una forma general, basándonos en la Victimología Evolutiva, se puede afirmar que los efectos de ser testigo de violencia son los característicos de la Psicopatología del Desarrollo, siendo propensas las víctimas a presentar problemas en su desarrollo evolutivo, emocional, cognitivo y social, que les impiden su bienestar y un progreso personal adecuado.

\section{TRANSMISIÓN GENERACIONAL DE LA VIOLENCIA: LA VIOLENCIA FAMILIAR COMO MODELO DE APRENDIZAJE}

Existen otros efectos, más a largo plazo, que se pueden asociar a la exposición de los niños a situaciones de violencia familiar. El más importante es que este tipo de situaciones constituye un modelo de aprendizaje de conductas violentas dentro del hogar, algo que junto a factores tales como los estilos de crianza punitivos, el abuso de sustancias y la presencia de trastornos de conducta en la adolescencia han demostrado poseer un papel relevante en el riesgo de ejercer violencia contra la pareja en la edad adulta ${ }^{3}$. Ehrensaflt, Cohen, Brown, Smailes, Chen y Jonson ${ }^{3}$ realizaron un estudio longitudinal sobre un período de 20 años en una muestra de 543 niños, concluyendo que entre los factores predictores del riesgo de ejercer violencia contra sus parejas se encontraban, en primer lugar, los trastornos de conducta, seguidos por la exposición a la violencia doméstica entre los padres y los sistemas de castigo basados en el poder.

La vivencia por parte de los niños de situaciones de violencia y abuso de poder cobra un significado 
crucial, puesto que las experiencias vividas en la infancia constituyen un factor de vital importancia para el posterior desarrollo y adaptación de la persona a su entorno. Los niños aprenden a definirse a sí mismos, a entender el mundo y cómo relacionarse con él a partir de lo que observan en su entorno más próximo. De este modo, la familia es considerada como el primer agente socializador del niño y el más determinante a la hora de la instauración de modelos apropiados de funcionamiento social. Las relaciones familiares, especialmente los estilos de crianza y la relación entre los padres, influyen sobre la capacidad del niño para la autorregularización de sus conductas y emociones, y sobre el significado que atribuirá a las relaciones interpersonales ${ }^{3}$.

Los niños que han experimentado alguna forma de rechazo parental o maltrato tienden a presentar sesgos atribucionales hostiles y aprenden a anticipar y a evitar las conductas de rechazo, generalizando esta anticipación a contextos interpersonales. Distintos estudios han constatado la alta probabilidad de que estos niños presenten déficits en el procesamiento de la información social ${ }^{3}$.

Por otra parte, los estilos parentales excesivamente punitivos o coercitivos pueden servir de modelo para la resolución coercitiva de los conflictos, que se generalizan desde las relaciones padres-hijos a las relaciones con los otros, facilitando el desarrollo de déficits en el funcionamiento interpersonal ${ }^{3}$. Estos primeros patrones de funcionamiento social, aprendidos y reforzados dentro de la familia, se aplican después a las interacciones con los iguales. De esta forma, los niños que exhiben estrategias interpersonales agresivas e inconsistentes con aquellas del grupo de iguales normativo tienen una alta probabilidad de no ser aceptados entre sus compañeros, con el consiguiente riesgo de aislamiento o de gravitar hacia grupos de iguales desviados o agresivos ${ }^{3}$. La pertenencia a estos grupos desviados en la adolescencia, junto con el reforzamiento parental continuado de estrategias interpersonales coercitivas o violentas, puede llegar a ser un importante obstáculo que limite las oportunidades de aprender a relacionarse con los otros de manera constructiva ${ }^{3}$.

Con la repetición, esos patrones de interacción y de resolución coercitiva de los conflictos se generalizan y se aplicarán, posteriormente, a las relaciones familiares y de pareja en la edad adulta ${ }^{3}$. A ello contribuye, por otro lado, las percepciones del carácter privado y relativamente impune del entorno familiar y la influencia de los estereotipos y creencias tradicionales acerca del uso y reparto del poder dentro de la familia.

La supervivencia intergeneracional de la violencia, y concretamente de la violencia de género, está determinada en gran medida por la influencia de factores de tipo cultural y educacional. Entre ellos, cobran especial relevancia los sistemas de valores que atribuyen una superioridad innata en los hombres respecto a las mujeres y la aceptación de la violencia como un medio válido para la resolución de conflictos. Tales sistemas de valores juegan un papel fundamental en el potencial de conductas sexistas y/o violentas en nuestros menores ${ }^{3}$.

Los hijos de mujeres maltratadas se ven expuestos no sólo a la influencia de factores de su entorno sociocultural, sino también a la propia experiencia de sufrir, bien como testigos o como víctimas, la violencia dentro de su entrono familiar. Así, los niños que crecen en hogares violentos aprenden e interiorizan una serie de creencias y valores violentos negativos sobre las relaciones familiares y sobre la legitimidad del uso de la violencia como método válido para la resolución de conflictos ${ }^{3}$.

\section{CONCLUSIONES Y PAUTAS ${ }^{7}$}

- La exposición a actos violentos contra la madre impacta directamente en los menores, con altas probabilidades de causarles daños severos en su desarrollo a corto y largo plazo. La historia del niño no es ajena a la historia de pareja. Por tanto, como parte de la evaluación, es necesario entrevistar a la madre, para disponer de toda la información posible sobre la violencia en la familia (por ejemplo, inicio y tipos de violencia, intensidad, frecuencia, formas de exposición de los menores, reacciones de los mismos a las agresiones). Según nos permita la edad de los niños, deberá explorarse su recuerdo y percepción de sucesos violentos durante y tras la convivencia.

- No todas las niñas y niños expuestos padecerán problemas clínicamente significativos, si bien se desconoce tanto la proporción de menores resilentes como los factores y moderadores que explican este hecho. La evaluación debe ser especialmente cuidadosa, tener en cuenta el diagnóstico diferencial, incluir múltiples informantes, escalas de adap- 
tación infantil y cuestionarios específicos. Además de entrevistas individuales con madre, menor y observaciones de la interacción de ambos, la información directamente aportada por la escuela y otros cuidadores es muy importante. Deben recogerse aspectos positivos, "fortalezas" del menor y su entorno, pero también aspectos que puedan perjudicar de alguna manera (por ejemplo, situación socioeconómica, problemas de salud de los principales cuidadores, relaciones entre hermanos).

- La violencia contra la mujer, y la consiguiente exposición a ella, sucede durante la convivencia y tras la separación. Para estas niñas y niños puede resultar muy difícil romper el secreto de la violencia en sus familias anteriores, pero no menos hablar sobre aspectos del régimen de visitas que pueden ser negativos. Las figuras paterna y materna deben ser abordadas respetando los sentimientos de los menores, y debemos estar atentos a signos que nos indiquen la posibilidad de manipulación de los niños, o la existencia de acoso tras la ruptura de pareja.

- Estas niñas y niños tienen un elevado riesgo de ser objeto de malos tratos físicos, psicológicos, sexuales y ser atendidos de forma negligente, en el contexto del maltratador. Por ello, otro elemento importante de la evaluación e intervención será la investigación de posibles indicadores de maltrato infantil.

- Las consecuencias para la salud mental de niños y niñas abarcan principalmente los trastornos de conducta, ansiedad y miedos, depresión, agresividad, baja autoestima y signos de trauma y estrés post-traumático. Esta no es una lista exclusiva, por lo cual debemos mantener un amplio espectro de dificultades en mente (por ejemplo, comportamientos regresivos, enuresis y encopresis, problemas del sueño, etc.), pero resultará útil disponer de experiencias en la evaluación e intervención en estos cuadros.

- Puede haber dificultades en el desarrollo de habilidades sociales, y creencias sobre la aceptabilidad de la violencia en las relaciones. Este es un aspecto importante no sólo de cara a futuras relaciones, sino también en tiempo presente, porque la relación con los iguales será fundamental, por ejemplo, para la recuperación de preadolescentes, adolescentes y jóvenes.

- El vínculo materno filial es objeto de ataques directos por parte del agresor, y a la vez un elemento sustancial en la recuperación de los menores. Parte importante de los objetivos será la evaluación del vínculo entre madre e hijos, y el restablecimiento ( $y$ a veces establecimiento) de una relación saludable entre ellos.

- Son necesarios estudios de calidad que faciliten el diseño y la implementación de programas de intervención precoz (protocolos de evaluación de riesgo).

- Es necesario sensibilizar a todos los profesionales en contacto con la infancia (salud, educación, policía, servicios sociales, puntos de encuentro) del problema del maltrato a menores y de las consecuencias de este maltrato en el desarrollo del niño en todas las esferas: física, cognitiva, afectiva, social y emocional.

\section{BIBLIOGRAFÍA}

1. Espinosa Bayal MA. Las hijas e hijos de mujeres maltratadas: consecuencias para su desarrollo e integración escolar. Instituto Vasco de la Mujer; 2004. Disponible en: http:// www.emakunde.es/images/upload/Angeles_Espinosa.pdf

2. Corsi J. Violencia familiar. Una mirada interdisciplinaria sobre un grave problema social. Buenos Aires: Ed. Paidós; 1994.

3. Patró Hernández R, Limiñana Gras RM. Víctimas de violencia familiar: Consecuencias psicológicas en hijos de mujeres maltratadas. Anales de Psicología. 2005; 21(1):117.

4. Lorente M, Lorente JA. Agresión a la mujer. Maltrato, violación y acoso. Granada: Comares SL; 1998.

5. Corbalan J, Patró R. Consecuencias psicológicas de la violencia familiar: mujeres maltratadas e hijos de hogares violentos. Conferencia impartida en II Jornadas sobre Mujer y Salud: Interacción de los contextos familiar y laboral. Murcia. Mayo 2003.

6. Pedreira Massa JL. La infancia en la familia con violencia: factores de riesgo y contenidos psico (pato)lógicos. Psiquiatría.com. 2003; 7(4).

7. Atenciano Jiménez B. Menores expuestos a Violencia contra la Pareja: Notas para una Práctica Clínica Basada en la Evidencia. Clínica y Salud. 2009; 20(3):261-72. 